\title{
Generalized Beamforming Design for Cooperative MIMO Multirelay Networks with Infinite Constraints and Imperfect CSI
}

\author{
Haiyang Yu, ${ }^{1,2}$ Wei Duan ${ }^{1},{ }^{1}$ Qiang Sun, ${ }^{1}$ Xia Wang, ${ }^{1}$ Jue Wang, \\ Jun Li, ${ }^{3}$ and Jaeho Choi ${ }^{2}{ }^{2}$ \\ ${ }^{1}$ School of Electronics and Information, Nantong University, Nantong 226019, China \\ ${ }^{2}$ Department of Electronics Engineering, CAIIT, Chonbuk National University, Chonju, Republic of Korea \\ ${ }^{3}$ School of Mechanical and Electrical Engineering, Guangzhou University, Guangzhou, China \\ Correspondence should be addressed to Wei Duan; sinder@ntu.edu.cn
}

Received 29 September 2017; Accepted 17 January 2018; Published 26 March 2018

Academic Editor: Abhishek K. Gupta

Copyright ( 2018 Haiyang Yu et al. This is an open access article distributed under the Creative Commons Attribution License, which permits unrestricted use, distribution, and reproduction in any medium, provided the original work is properly cited.

\begin{abstract}
A generalized base station-relay-user equipment (BS-Relay-UE) beamforming design is investigated for a cooperative multipleinput multiple-output (MIMO) multirelay networks with imperfect channel state information (CSI). In order to minimize the worstcase mean square error (MSE) which is subject to a semi-infinite (SI) relay power constraints, a generalized optimal beamforming structure for the relay amplifying matrix is effectively proposed, and then the SI relay power constraints are converted into linear matrix inequalities (LMIs) version. In such conversion, the objective problem recasts as a decoupled biconvex semidefinite programming (SDP) one which can be efficiently solved by the proposed alternating algorithm. The system performance has been verified in terms of worst-case MSE using a set of qualitative analyses. The results show us that the proposed beamforming method outperforms the conventional schemes and can also effectively reduce the computational complexity when it is compared to the cutting-set schemes and also to the nonrobust ones.
\end{abstract}

\section{Introduction}

Recently, cooperative multi-input multi-output (MIMO) relay network approaches are popularized to increase the system capacity and to improve the transmission reliability by leveraging spatial diversity. Since the channel estimation is important to the wireless communications, the authors in [1] investigated the main challenges faced by high-dimensional channel state information (CSI) acquisition in massive MIMO systems which was first shown that the narrow angular spread or the spatial sparsity is crucial for all low-rank approaches. Cooperative relay network technology has attracted significant interests due to the superior spectral efficiency. Various cooperative relaying schemes have been proposed, such as denoise-and-forward (DNF) [2-4], decode-and-forward (DF) [5, 6], and amplify-andforward (AF) [7-12]. Specifically, the spatially correlated fading channels are considered in [13], which is more practical and challenging. Training designs for estimation of spatially correlated multiple-input multiple-output (MIMO) AF twoway multirelay channels are studied in [13], where an optimal training structure is initially derived to minimize total mean square error (MSE) of the channel estimation.

Considering inaccurate channel estimation and feedback delay, the perfect channel state information (CSI), which are proposed in the above works, is usually hard to obtain in practice. In order to circumvent these problems, by taking into account the channel uncertainties, the imperfect CSI scenario has been studied in [7, 14-20]. The authors in [14] considered robust transceiver design in a multiuser MIMO multirelay cognitive radio network with interference power constraints and individual transmission power constraints. In [15], the authors have investigated the global optimal transceiver design in the MIMO link under a deterministic CSI uncertainty model. Specifically, a robust minimum mean-square-error (MMSE-) based beamforming scheme for 


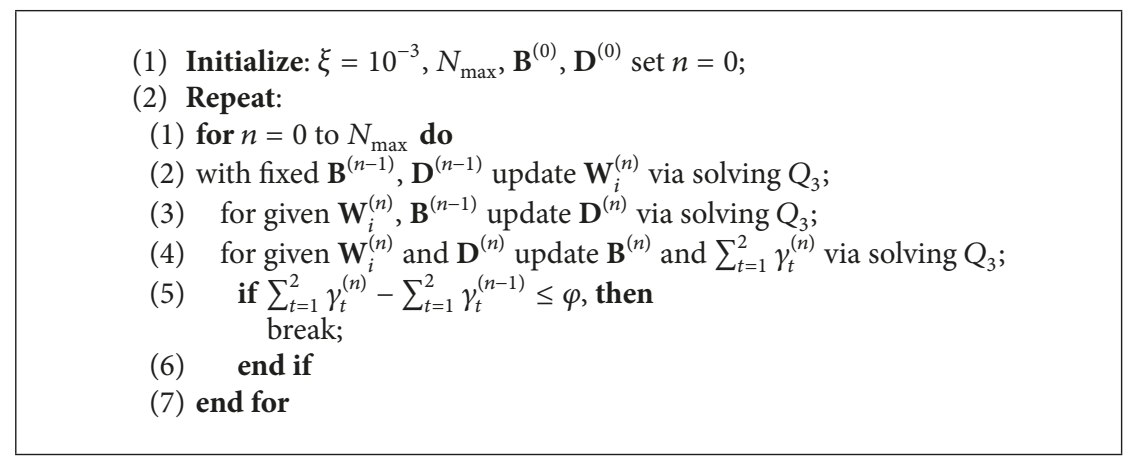

Algorithm 1: The proposed generalized BS-R-UE robust beamforming scheme.

an amplify-and-forwarding multiantenna relay network is considered in [16]. Here, the authors divided the original problem into subproblems which are formulated into a convex optimization framework. By taking the imperfect channel state information into consideration, in [17], the robust AF-MIMO transceiver optimization is developed in order to combat correlated channel uncertainties, where the matrix-form conjugate gradient (MCG) method is used for optimization. Moreover, the robust joint optimization of the relay weights and the input covariance matrix of jamming signals for secrecy rate maximization is addressed in [18]. In [19], the authors investigated the worst-case robust relay precoder optimization for multiantenna AF relaying in the presence of either deterministic additive or multiplicative channel uncertainties. Specifically, the authors in $[7,20]$ have considered a two-way relay network consisting of multiple pairs of single-antenna users and distributed, multiple relays.

Furthermore, in [21-26], the generalization of S-Lemma to complex-valued variables and multiple semi-infinite (SI) constraints are presented. They have investigated iterative algorithms based on alternating convex search (ACS) to devise practical algorithms for solving the semi-infinite problems. In addition, in [26], the authors investigated a robust transceiver design for downlink multiuser MIMO AF-relay systems with norm-bounded channel uncertainties presenting that the cutting-set method proposed improves the performance of the worst-case MSE.

Since that, for the multiple relays scheme, not only the performance of the capacity outperforms that of the single relay one, but also the multiple relays scheme is more practical and challenging for the wireless communication scenarios. The downlink network considered here consists of a base station (BS), multiple relays, and user equipment (UE). We have named this particular AF system base BS-Relays-UE beamforming. The main contributions of this paper are summarized in the following:

(i) We comprehensively investigate a generalized beamforming design for a cooperative MIMO multirelay networks with imperfect CSI. In the proposed scheme, the joint optimal design considers the precoding matrix at the BS, the beamforming matrix at the relays and the receiving beamforming matrix at the UE with multiple relays, which is general and practical.

(ii) Since the considered worst-case MSE optimization problem is not only nonconvex but also subject to the semi-infinite relay power constraints, a generalized optimal beamforming structure of the relay amplifying matrix is investigated. In addition, the SI relay power constraints are converted into linear matrix inequalities (LMIs). By this way, the objective problem recasts as a decoupled biconvex semidefinite programming (SDP) one, which can be efficiently solved by our proposed alternating algorithm.

(iii) By means of the numerical results, the proposed beamforming design significantly reduces the computational cost and improves the performance in terms of the worst-case MSE compared with the nonrobust and cutting-set cases.

In the following, a system model for the proposed cooperative MIMO multirelay networks is presented in Section 2 and a generalized BS-Relay-UE beamforming design is described in Section 3 (see Algorithm 1). In Section 4, the performance of the proposed system is evaluated using qualitative analysis and the numerical results are provided. Finally, the conclusions are made in Section 5.

Notations. $\mathbf{A}^{T}, \mathbf{A}^{\dagger}, \operatorname{tr}(\mathbf{A}),\|\mathbf{A}\|_{2}$, and $\|\mathbf{A}\|_{F}$ denote the transpose, conjugate transpose, trace, Euclidean norm, and Frobenius norm of a matrix $\mathbf{A}$, respectively. $\mathfrak{R}(\cdot)$ and $\mathfrak{\Im}(\cdot)$ are operators for taking the real part and imaginary part of a complex-valued matrix, respectively. The field of $m \times n$ dimensional complex-valued matrix is denoted by $\mathbb{C}^{M \times M} . I_{N}$ is an $N \times N$ identity matrix. $\odot$ and $\otimes$ are the operators for computing Hadamard and Kronecker products.

\section{System Model}

In this section, an amplify-and-forward relaying system model is described. In the proposed system, users are away from a base station and they are isolated and out of the BS coverage. In other words, the direct link between BS and UE does not exist so that UE can get a connection to the BS only via relays in order to achieve any signal receptions. To be more 


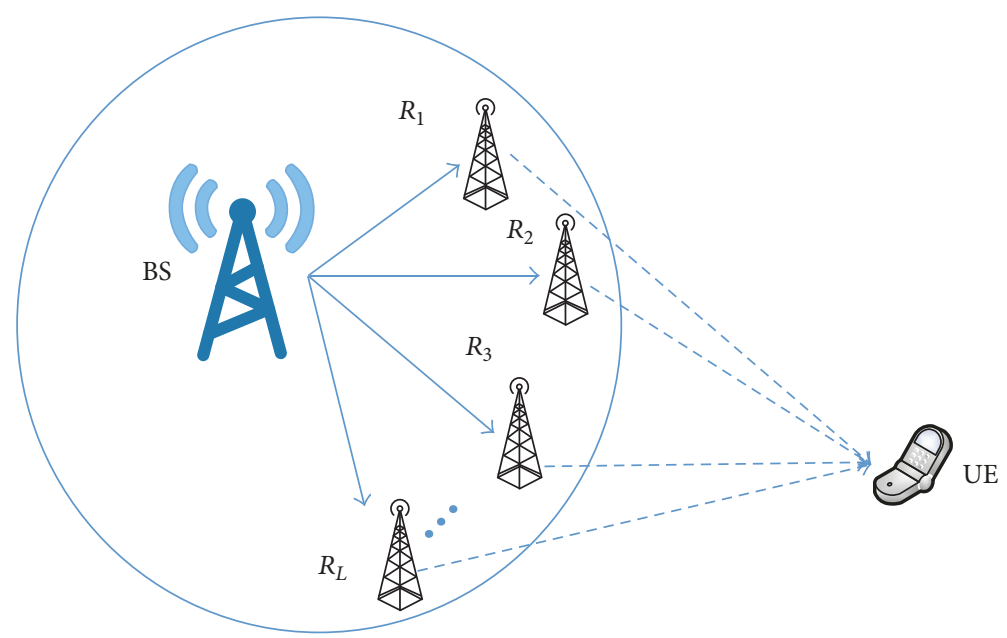

FIgURe 1: Cooperative MIMO multirelay networks.

specific, the proposed multirelay network consists of one BS, $L$ relays, and one UE as shown in Figure 1. In this network, communications are performed using a half-duplex mode. It is further assumed that the BS and UE are equipped with $M$ antennas while each relay node is with $N$ antennas.

Now, concerning the communication channels related to the proposed network, there are two of them. The one is the group of estimated communication channels $\mathbf{G}_{i} \in \mathbb{C}^{N \times M}$, which are from the BS to $L$ relays $R_{i}$, for $i \in\{1, \ldots, L\}$; the other one is another group of communication channels $\mathbf{F}_{i} \in \mathbb{C}^{M \times N}$, which are from $L$ relays $R_{i}$ to UE. We have also considered $N \geq M$ to satisfy the decodeable condition.

It is assumed that the BS transmits the signals to the UE using two consecutive time slots. At the first time slot, after being linearly processed by the matrix $\mathbf{B} \in \mathbb{C}^{M \times M}$, which is constrained by $\operatorname{tr}\left\{\mathbf{B B}^{H}\right\} \leq P_{t}$, the information data $\mathbf{x}=\left[x_{1}, \ldots, x_{m}\right]^{T}$ with unit power is transmitted to the relay nodes. Therefore, the received signal at $R_{i}$ can be expressed as

$$
\mathbf{y}_{R_{i}}=\mathbf{G}_{i} \mathbf{B x}+\mathbf{n}_{R_{i}},
$$

where $\mathbf{n}_{R_{i}} \sim \mathscr{C} \mathscr{N}\left(0, \sigma_{R_{i}}^{2} \mathbf{I}_{N}\right)$ represents the additive white Gaussian noise (AWGN) vector with zero mean and variance $\sigma_{R_{i}}^{2}$ at the relay node $R_{i}$.

At the second time slot, the relay node $R_{i}$ linearly amplifies $\mathbf{y}_{R_{i}}$ with an $N \times N$ beamforming matrix $\mathbf{W}_{i}$ and then broadcasts the amplified signal vector $\mathbf{x}_{R_{i}}$ to the UE, which results in

$$
\mathbf{x}_{R_{i}}=\mathbf{W}_{i} \mathbf{y}_{R_{i}} .
$$

Therefore, the transmission power used by the relay node $R_{i}$ is given by

$$
\begin{aligned}
\mathbf{E}\left\{\left\|\mathbf{x}_{R_{i}}\right\|_{2}^{2}\right\} & =\operatorname{tr}\left\{\mathbf{W}_{i} \mathbf{y}_{R_{i}} \mathbf{y}_{R_{i}}^{\dagger} \mathbf{W}_{i}^{\dagger}\right\} \\
& =\operatorname{tr}\left\{\mathbf{W}_{i}\left(\mathbf{G}_{i} \mathbf{B} \mathbf{B}^{\dagger} \mathbf{G}_{i}^{\dagger}+\sigma_{R_{i}}^{2} \mathbf{I}_{N}\right) \mathbf{W}_{i}^{\dagger}\right\} .
\end{aligned}
$$

At the UE, the received data is linearly utilized by using the receiving beamforming matrix $\mathbf{D} \in \mathbb{C}^{M \times M}$ with $\operatorname{tr}\left\{\mathbf{D D}^{\dagger}\right\} \leq$
$P_{d}$, where $\leq P_{d}$ is a slack value. The received signal vectors at the UE can be finally denoted as

$$
\mathbf{y}=\mathbf{D}\left(\sum_{i=1}^{L} \mathbf{F}_{i} \mathbf{W}_{i} \mathbf{G}_{i} \mathbf{B} \mathbf{x}+\sum_{i=1}^{L} \mathbf{F}_{i} \mathbf{W}_{i} \mathbf{n}_{R_{i}}+\mathbf{n}_{D}\right),
$$

where $\mathbf{n}_{D}$ is the received noise vector at the UE with mean zero and variance $\sigma_{D}^{2}$.

By taking into account the estimation error, the CSI is considered partially known at each node. With this consideration, the actual channel coefficients of the links follow that

$$
\begin{aligned}
& \mathbf{G}_{i} \triangleq\left\{\widetilde{\mathbf{G}}_{i}+\Delta_{\mathbf{G}_{i}}\right\}, \\
& \mathbf{F}_{i} \triangleq\left\{\widetilde{\mathbf{F}}_{i}+\Delta_{\mathbf{F}_{i}}\right\}
\end{aligned}
$$

with $\left\{\Delta_{\mathbf{G}_{i}}, \Delta_{\mathbf{F}_{i}}\right\}$ as the channel uncertainties. For simplicity, the channel uncertainties are assumed to be norm-bounded errors (NBEs) [24] as

$$
\begin{gathered}
\left\|\Delta_{\mathbf{G}_{i}}\right\| \leq \alpha_{i}, \\
\left\|\Delta_{\mathbf{F}_{i}}\right\| \leq \beta_{i},
\end{gathered}
$$

where $0 \leq\left\{\alpha_{i}, \beta_{i}\right\} \ll 1$.

With this observation, the worst-case MSE at the UE node can be obtained as

$$
\begin{gathered}
\varphi \triangleq\left\|\sum_{i=1}^{L} \mathbf{D}\left(\widetilde{\mathbf{F}}_{i}+\Delta_{\mathbf{F}_{i}}\right) \mathbf{W}_{i}\left(\widetilde{\mathbf{G}}_{i}+\Delta_{\mathbf{G}_{i}}\right) \mathbf{B}-\mathbf{I}_{M}\right\|_{F}^{2} \\
+\sigma_{R_{i}}^{2}\left\|\sum_{i=1}^{L} \mathbf{D}\left(\widetilde{\mathbf{F}}_{i}+\Delta_{\mathbf{F}_{i}}\right) \mathbf{W}_{i}\right\|_{F}^{2}+\sigma_{D}^{2}\|\mathbf{D}\|_{F}^{2} \\
\leq \underbrace{\left\|\sum_{i=1}^{L} \mathbf{D}\left(\widetilde{\mathbf{F}}_{i}+\Delta_{\mathbf{F}_{i}}\right) \mathbf{W}_{i}\left(\widetilde{\mathbf{G}}_{i}+\Delta_{\mathbf{G}_{i}}\right) \mathbf{B}-\mathbf{I}_{M}\right\|_{F}^{2}}_{\mathscr{M}_{1}}
\end{gathered}
$$




$$
\begin{aligned}
& +\underbrace{+\sigma_{R_{i}}^{2}\left\|\sum_{i=1}^{L} \mathbf{D}\left(\widetilde{\mathbf{F}}_{i}+\Delta_{\mathbf{F}_{i}}\right) \mathbf{W}_{i}\right\|_{E}^{2}}_{\mathscr{M}_{2}}+\sigma_{D}^{2} P_{d} \\
& =\mathscr{M}_{1}+\mathscr{M}_{2}+\sigma_{D}^{2} P_{d} .
\end{aligned}
$$

Since $\mathscr{M}_{1}+\mathscr{M}_{2} \geq \varphi-\sigma_{D}^{2} P_{d}$, after introducing the slack value $\widehat{\varphi}=\varphi-\sigma_{D}^{2} P_{d}$, the objective problem is to minimize the worstcase MSE subjects to the BS and the relay transmission power constraints which can be formulated as follows:

$$
\begin{aligned}
Q_{1}: \min _{\mathbf{B}, \mathbf{W}_{i}, \mathbf{D}} & \mathscr{M}_{1}+\mathscr{M}_{2} \\
\text { s.t. } & \left\|\mathbf{x}_{R_{i}}\right\|^{2} \leq P_{R_{i}}, \\
& \operatorname{tr}\left\{\mathbf{B B}^{\dagger}\right\} \leq P_{t}, \\
& \operatorname{tr}\left\{\mathbf{D D}^{\dagger}\right\} \leq P_{d}, \\
& \widehat{\varphi} \leq \mathscr{M}_{1}+\mathscr{M}_{2},
\end{aligned}
$$

where $P_{R_{i}}$ denotes the power constraint at the relay node. In problem $Q_{1}$, it is clear that not only the objective problem $\mathscr{M}_{1}+\mathscr{M}_{2}$ is nonconvex, but also the semi-infinite expressions of the optimal $\mathbf{W}_{i}, \mathbf{D}$, and $\mathbf{B}$ are intractable. In particular, the globally optimal solution is difficult to be obtained; in this paper, to efficiently solve $Q_{1}$, a biconvex SDP is proposed to obtain the suboptimal solution of the worst-case MSE for our proposed scheme.

$$
\mathbf{F}_{i}^{\star} \mathbf{W}_{i} \mathbf{G}_{i}^{\star}=\boldsymbol{\Pi}_{i}\left[\left[\mathbf{F}_{i}^{\diamond}\right]_{M \times M} \mathbf{0}_{M \times(N-M)}\right]\left[\begin{array}{l}
{\left[\mathbf{W}_{i}^{\diamond}\right]_{M \times M}} \\
\mathbf{Y}_{(N-M) \times M}
\end{array}\right.
$$

In this manner, we have

$$
\begin{aligned}
& M_{1}+\mathscr{M}_{2} \\
& =\left\|\sum_{i=1}^{L}\left(\boldsymbol{\Pi}_{i} \mathbf{F}_{i}^{\diamond} \mathbf{W}_{i}^{\diamond} \mathbf{G}_{i}^{\diamond} \mathbf{\Omega}_{i}^{\dagger}+\boldsymbol{\Phi}_{i}\right)-\mathbf{I}_{M}\right\|_{F}^{2} \\
& +\sigma_{R_{i}}^{2}\left\|\sum_{i=1}^{L}\left(\boldsymbol{\Pi}_{i}\left[\begin{array}{lll}
\mathbf{F}_{i}^{\curlyvee} \mathbf{W}_{i}^{\diamond} & \mathbf{F}_{i}^{\diamond} \mathbf{X}
\end{array}\right] \mathbf{U}_{i}^{\dagger}+\Psi_{i}\right)\right\|_{F}^{2},
\end{aligned}
$$

where $\Psi_{i}=\mathbf{D} \Delta_{\mathbf{F}_{i}} \mathbf{V}_{i} \mathbf{C}_{i} \mathbf{U}_{i}^{\dagger}$ and $\boldsymbol{\Phi}_{i}=\mathbf{D} \Delta_{\mathbf{F}_{i}} \mathbf{V}_{i} \mathbf{C}_{i} \boldsymbol{\Gamma}_{i}+$ $\boldsymbol{\Sigma}_{i} \mathbf{C}_{i} \mathbf{U}_{i}^{\dagger} \Delta_{\mathbf{G}_{i}}$ B. It is worth noting that the term $\mathbf{D} \Delta_{\mathbf{F}_{i}} \mathbf{V}_{i} \mathbf{C}_{i} \mathbf{U}_{i}^{\dagger} \Delta_{\mathbf{G}_{i}} \mathbf{B}$ becomes 0 , because if we retain this term in $\boldsymbol{\Phi}_{i}$, when calculating the MSE, it will result in some terms involving $3 \mathrm{rd}$ and 4 th order of channel uncertainties which are negligible.

\section{Generalized BS-Relay-UE Beamforming Design}

3.1. The Optimal Relay Beamforming Design. With the fixed BS and UE beamforming matrices $\mathbf{B}$ and $\mathbf{D}$, suppose the singular value decomposition (SVD) of $\mathbf{F}_{i}^{\star}=\mathbf{D} \widetilde{\mathbf{F}}_{i}$ and $\mathbf{G}_{i}^{\star}=$ $\widetilde{\mathbf{G}}_{i} \mathbf{B}$ as follows:

$$
\begin{aligned}
& \mathbf{F}_{i}^{\star}=\boldsymbol{\Pi}_{i}\left[\left[\mathbf{F}_{i}^{\diamond}\right]_{M \times M} \mathbf{0}_{M \times(N-M)}\right] \mathbf{V}_{i}^{\dagger} \triangleq \boldsymbol{\Pi}_{i} \Sigma_{i} \mathbf{V}_{i}^{\dagger}, \\
& \mathbf{G}_{i}^{\star}=\mathbf{U}_{i}\left[\begin{array}{l}
{\left[\mathbf{G}_{i}^{\diamond}\right]_{M \times M}} \\
\mathbf{0}_{(N-M) \times M}
\end{array}\right] \boldsymbol{\Omega}_{i}^{\dagger} \triangleq \mathbf{U}_{i} \boldsymbol{\Gamma}_{i} \boldsymbol{\Omega}_{i}^{\dagger},
\end{aligned}
$$

where $\mathbf{V}_{i} \in \mathbb{C}^{N \times N}, \mathbf{U}_{i} \in \mathbb{C}^{N \times N}, \boldsymbol{\Pi}_{i} \in \mathbb{C}^{M \times M}$, and $\boldsymbol{\Omega}_{i} \in \mathbb{C}^{M \times M}$ are unitary matrices.

Theorem 1. Using the SVDs in (9), the optimal relay beamforming matrix as the solution to the objective problem $Q_{1}$ can be obtained as

$$
\mathbf{W}_{i}=\mathbf{V}_{i} \mathbf{C}_{i} \mathbf{U}_{i}^{\dagger}
$$

where $\mathbf{C}_{i} \in \mathbb{C}^{N \times N}$ is a matrix to be determined.

Proof. The proof is similar to $[8,25]$.

Without loss of generality, $\mathbf{C}_{i}$ can be further partitioned as follows:

$$
\mathbf{C}_{i}=\left[\begin{array}{cc}
{\left[\mathbf{W}_{i}^{\diamond}\right]_{M \times M}} & \mathbf{X}_{M \times(N-M)} \\
\mathbf{Y}_{(N-M) \times M} & \mathbf{Z}_{(N-M) \times(N-M)}
\end{array}\right] .
$$

From (9), (11) and Theorem 1, one can have

$$
\left.\mathbf{X}_{M \times(N-M)}\right]\left[\begin{array}{c}
{\left[\mathbf{G}_{i}^{\diamond}\right]_{M \times M}} \\
\mathbf{Z}_{(N-M) \times(N-M)}
\end{array}\right] \mathbf{\Omega}_{i}^{\dagger}=\boldsymbol{\Pi}_{i} \mathbf{F}_{i}^{\diamond} \mathbf{W}_{i}^{\diamond} \mathbf{G}_{i}^{\diamond} \mathbf{\Omega}_{i}^{\dagger}
$$

Using $\|\mathbf{A}+\mathbf{B}\| \leq\|\mathbf{A}\|+\|\mathbf{B}\|$ and $\|\mathbf{A B}\| \leq\|\mathbf{A}\|\|\mathbf{B}\|$, from (13), we have

$$
\begin{aligned}
& \mathscr{M}_{1} \leq\left\|\sum_{i=1}^{L} \boldsymbol{\Pi}_{i} \mathbf{F}_{i}^{\curlyvee} \mathbf{W}_{i}^{\curlyvee} \mathbf{G}_{i}^{\diamond} \mathbf{\Omega}_{i}-\mathbf{I}_{M}\right\|_{F}^{2}+\sum_{i=1}^{L}\left\|\Phi_{i}\right\|_{F}^{2} \\
& \leq \sum_{i=1}^{L}\left(P_{d} P_{t} \alpha_{i} \beta_{i}+P_{d} \beta_{i}\left\|\mathbf{G}_{i}^{\diamond}\right\|_{F}^{2}+P_{t} \alpha_{i}\left\|\mathbf{F}_{i}^{\diamond}\right\|_{F}^{2}\right) \\
& \cdot\left\|\left[\begin{array}{ll}
{\left[\mathbf{W}_{i}^{\diamond}\right]_{M \times M}} & \mathbf{X}_{M \times(N-M)} \\
\mathbf{Y}_{(N-M) \times M} & \mathbf{Z}_{(N-M) \times(N-M)}
\end{array}\right]\right\|_{F}^{2} \\
& +\left\|\sum_{i=1}^{L} \mathbf{F}_{i}^{\diamond} \mathbf{W}_{i}^{\diamond} \mathbf{G}_{i}^{\diamond}-\boldsymbol{\Pi}_{i}^{\dagger} \mathbf{\Omega}_{i}\right\|_{F}^{2} \\
& \mathscr{M}_{2} \leq \sigma_{R_{i}}^{2}\left\|\sum_{i=1}^{L} \boldsymbol{\Pi}_{i}\left[\begin{array}{lll}
\mathbf{F}_{i}^{\diamond} \mathbf{W}_{i}^{\diamond} & \mathbf{F}_{i}^{\diamond} \mathbf{X}
\end{array}\right]\right\|_{F}^{2}+\sigma_{R_{i}}^{2} \sum_{i=1}^{L}\left\|\Psi_{i}\right\|_{F}^{2}
\end{aligned}
$$




$$
\begin{aligned}
& \leq \sigma_{R_{i}}^{2}\left\|\sum_{i=1}^{L}\left[\begin{array}{lll}
\mathbf{F}_{i}^{\curlyvee} \mathbf{W}_{i}^{\curlyvee} & \mathbf{F}_{i}^{\curlyvee} \mathbf{X}
\end{array}\right]\right\|_{F}^{2}+\sigma_{R_{i}}^{2} P_{d} \sum_{i=1}^{L} \beta_{i} \\
& \left\|\left[\begin{array}{ll}
{\left[\mathbf{W}_{i}^{\diamond}\right]_{M \times M}} & \mathbf{X}_{M \times(N-M)} \\
\mathbf{Y}_{(N-M) \times M} & \mathbf{Z}_{(N-M) \times(N-M)}
\end{array}\right]\right\|_{F}^{2} .
\end{aligned}
$$

Since the channel uncertainty $\Delta_{\mathbf{G}_{i}}$ can be further partitioned,

$$
\Delta_{\mathbf{G}_{i}}=\left[\begin{array}{c}
{\left[\Delta_{\mathbf{G}_{i}}^{\diamond}\right]_{M \times M}} \\
{\left[\Delta_{\mathbf{G}_{i}}^{b}\right]_{M \times(N-M)}}
\end{array}\right],
$$

upon substituting (10), (11), and (15) into the relay power constraint (3), we have

$$
\begin{aligned}
\mathbf{E}\left\{\left\|\mathbf{x}_{R_{i}}\right\|_{2}^{2}\right\} & \\
= & \operatorname{tr}\left\{\mathbf{W}_{i}^{\diamond}\left(\mathbf{G}_{i}^{\sharp} \mathbf{B}\left(\mathbf{G}_{i}^{\sharp} \mathbf{B}\right)^{\dagger}+\sigma_{R_{i}}^{2} \mathbf{I}_{M}\right)\left(\mathbf{W}_{i}^{\diamond}\right)^{\dagger}+\mathbf{X} \mathbf{X}^{\dagger}\right\} \\
& +\operatorname{tr}\left\{\mathbf{Z} \mathbf{Z}^{\dagger}+\mathbf{Y}\left(\mathbf{G}_{i}^{\sharp} \mathbf{B}\left(\mathbf{G}_{i}^{\sharp} \mathbf{B}\right)^{\dagger}+\sigma_{R_{i}}^{2} \mathbf{I}_{M}\right) \mathbf{Y}^{\dagger}\right\},
\end{aligned}
$$

where $\mathbf{G}_{i}^{\sharp}=\mathbf{G}_{i}^{\diamond}+\Delta_{\mathbf{G}_{i}}^{\diamond}$. Synthesizing (14) and (16), it is clear that, for any feasible $\mathbf{C}_{i}$ with $\{\mathbf{X}, \mathbf{Y}, \mathbf{Z}\} \neq \mathbf{0}$, one can always find

$$
\mathbf{C}_{i}^{\prime}=\left[\begin{array}{cc}
{\left[\mathbf{W}_{i}^{\curlyvee}\right]_{M \times M}} & \mathbf{0}_{M \times(N-M)} \\
\mathbf{0}_{(N-M) \times M} & \mathbf{0}_{(N-M) \times(N-M)}
\end{array}\right],
$$

which can achieve the smaller relay power constraint and MSE. Thus, we conclude that if and only if $\mathbf{X}=\mathbf{Y}=\mathbf{Z}=\mathbf{0}$, the objective problem $\left\{\min \mathscr{M}_{1}+\mathscr{M}_{2}\right\}$ has the optimal solution. By this way, the optimal expression of $\mathbf{W}_{i}$ can be denoted as

$$
\mathbf{W}_{i}^{\sharp}=\mathbf{V}_{i}\left[\begin{array}{cc}
{\left[\mathbf{W}_{i}^{\diamond}\right]_{M \times M}} & \mathbf{0}_{M \times(N-M)} \\
\mathbf{0}_{(N-M) \times M} & \mathbf{0}_{(N-M) \times(N-M)}
\end{array}\right] \mathbf{U}_{i}^{\dagger} .
$$

3.2. The Joint Source and Relay Beamforming Design. From (14), it is clear that, with fixed $\mathbf{W}_{i}^{\diamond}$, the objective problem $\left\{\min \left(\mathscr{M}_{1}+\mathscr{M}_{2}\right)\right\}$ is equivalent to the problem:

$$
\min \left\|\sum_{i=1}^{L} \boldsymbol{\Phi}_{i}\right\|_{F}^{2}+\left\|\sum_{i=1}^{L} \boldsymbol{\Psi}_{i}\right\|_{F}^{2} .
$$

Therefore, similar to [22], by introducing the auxiliary optimization variable $\gamma_{t}$, for $t=1,2$, problem (19) can be recast in the epigraph form [27] as $\left\{\min \left(\gamma_{1}+\gamma_{2}\right)\right\}$ which subjects to $\left\|\sum_{i=1}^{L} \boldsymbol{\Phi}_{i}\right\|_{F}^{2} \leq \gamma_{1},\left\|\sum_{i=1}^{L} \boldsymbol{\Psi}_{i}\right\|_{F}^{2} \leq \gamma_{2}$. In this manner, the objective problem $Q_{1}$ can be equivalently converted into the problem $Q_{2}$ as follows:

$$
\begin{array}{ll}
Q_{2}: \min _{\mathbf{B}, \mathbf{D}} & \gamma_{1}+\gamma_{2} \\
\text { s.t. } & \left\|\mathbf{x}_{R_{i}}\right\|^{2} \leq P_{R_{i}}, \\
& \operatorname{tr}\left\{\mathbf{B B}^{\dagger}\right\} \leq P_{t}, \\
& \operatorname{tr}\left\{\mathbf{D D}^{\dagger}\right\} \leq P_{d}, \\
& \left\|\sum_{i=1}^{L} \boldsymbol{\Phi}_{i}\right\|_{F}^{2} \leq \gamma_{1}, \\
& \left\|\sum_{i=1}^{L} \boldsymbol{\Psi}_{i}\right\|_{F}^{2} \leq \gamma_{2} .
\end{array}
$$

Using the identities $\|\mathbf{A}\|=\|\operatorname{vec}[\mathbf{A}]\|$ and $\operatorname{vec}[\mathbf{A B C}]=\left(\mathbf{C}^{T} \otimes\right.$ $\mathbf{A})$ vec $[\mathbf{B}]$, for any given matrix $\mathbf{A}, \mathbf{B}$ and $\mathbf{C}$, for the constraint $\left\|\sum_{i=1}^{L} \boldsymbol{\Phi}_{i}\right\|_{F}^{2} \leq \gamma_{1}$, we have

$$
\begin{aligned}
& \left\|\sum_{i=1}^{L} \boldsymbol{\Phi}_{i}\right\|_{F}^{2}=\left\|\sum_{i=1}^{L} \operatorname{vec}\left[\boldsymbol{\Phi}_{i}\right]\right\|_{F}^{2} \\
& =\| \sum_{i=1}^{L}\left(\left(\mathbf{V}_{i} \mathbf{C}_{i} \boldsymbol{\Gamma}_{i}\right)^{T} \otimes \mathbf{D}\right) \operatorname{vec}\left[\Delta_{\mathbf{F}_{i}}\right]+\sum_{i=1}^{L} \mathbf{B}^{T} \\
& \otimes\left(\boldsymbol{\Sigma}_{i} \mathbf{C}_{i} \mathbf{U}_{i}^{\dagger}\right) \operatorname{vec}\left[\Delta_{\mathbf{G}_{i}}\right] \|_{F}^{2}
\end{aligned}
$$

Further assuming $\sum_{i=1}^{L} \operatorname{vec}\left[\boldsymbol{\Phi}_{i}\right]=\aleph_{i}$, the constraint $\left\|\sum_{i=1}^{L} \boldsymbol{\Phi}_{i}\right\|_{F}^{2} \leq \gamma_{1}$ can be represented in the term of the following LMI form as

$$
\left[\begin{array}{cc}
\gamma_{1} & \aleph_{i}^{\dagger} \\
\aleph_{i} & \mathbf{I}
\end{array}\right] \geq 0
$$

By employing S-Lemma [22], (22) can be recast as

$$
\left[\begin{array}{ccccc}
\gamma_{1} & \mathbf{0}_{1 \times M N} & \mathbf{0}_{1 \times M N} & \cdots & \mathbf{0}_{1 \times M N} \\
\mathbf{0}_{M N \times 1} & \mathbf{I}_{M N} & -\boldsymbol{\Xi}_{1}^{\dagger} & \cdots & -\boldsymbol{\Xi}_{2 L}^{\dagger} \\
\mathbf{0}_{M N \times 1} & -\boldsymbol{\Xi}_{1} & \epsilon_{1} \mathbf{I}_{M N} & \cdots & \mathbf{0} \\
\vdots & \vdots & \vdots & \ddots & \vdots \\
\mathbf{0}_{M N \times 1} & -\boldsymbol{\Xi}_{2 L} & \mathbf{0} & \cdots & \epsilon_{2 L} \mathbf{I}_{M N}
\end{array}\right] \geq 0
$$

where $\epsilon_{i}$, for $i \in\{1, \ldots, 2 L\}$, is a slack variable, and

$$
\boldsymbol{\Xi}_{j}= \begin{cases}{\left[\alpha_{i} \mathbf{M}_{G_{i}}, \mathbf{0}_{M N \times M(N-M)}\right],} & j=1, \ldots, L, \\
{\left[\begin{array}{c}
\beta_{i} \mathbf{M}_{F_{i}} \\
\mathbf{0}_{M N \times M(N-M)}
\end{array}\right],} & j=L+1, \ldots, 2 L,\end{cases}
$$


where

$$
\begin{gathered}
\mathbf{M}_{F_{i}}=\left(\mathbf{V}_{i} \mathbf{C}_{i} \boldsymbol{\Gamma}_{i}\right)^{T} \otimes \mathbf{D}, \\
\mathbf{M}_{G_{i}}=\mathbf{B}^{T} \otimes\left(\boldsymbol{\Sigma}_{i} \mathbf{C}_{i} \mathbf{U}_{i}^{\dagger}\right) .
\end{gathered}
$$

On the other hand, similarly, letting $\Theta_{F_{i}}=\left(\mathbf{V}_{i} \mathbf{C}_{i} \mathbf{U}_{i}^{\dagger}\right)^{T} \otimes \mathbf{D}$, the constraint $\left\|\sum_{i=1}^{L} \Psi_{i}\right\|_{F}^{2}$ can be equivalently converted into the LMI version as follows:

$$
\left[\begin{array}{ccccc}
\gamma_{2} & \mathbf{0}_{1 \times M N} & \mathbf{0}_{1 \times M N} & \cdots & \mathbf{0}_{1 \times M N} \\
\mathbf{0}_{M N \times 1} & \mathbf{I}_{M N} & -\boldsymbol{\Theta}_{F_{1}}^{\dagger} & \cdots & -\boldsymbol{\Theta}_{F_{L}}^{\dagger} \\
\mathbf{0}_{M N \times 1} & -\boldsymbol{\Theta}_{F_{i}} & \varsigma_{1} \mathbf{I}_{M N} & \cdots & \mathbf{0} \\
\vdots & \vdots & \vdots & \ddots & \vdots \\
\mathbf{0}_{M N \times 1} & -\boldsymbol{\Theta}_{F_{L}} & \mathbf{0} & \cdots & \varsigma_{L} \mathbf{I}_{M N}
\end{array}\right] \geq 0,
$$

where $\varsigma_{i}$ is a slack variable. In this observation, (23) and (26) represented the equivalent SDP formulation of the constraints $\left\|\sum_{i=1}^{L} \boldsymbol{\Phi}_{i}\right\|_{F}^{2} \leq \gamma_{1}$ and $\left\|\sum_{i=1}^{L} \boldsymbol{\Psi}_{i}\right\|_{F}^{2}$ which are affine in the channel uncertainties.

For the relay power constraint, after introducing the slack variable $P^{\diamond}$, it can be decoupled into two parts as follows:

$$
\begin{array}{r}
\overline{P^{\diamond}}+\bar{\sigma}_{R_{i}}^{2}\left\|\mathbf{W}_{i}^{\diamond}\right\|_{F}^{2} \leq \bar{P}_{R_{i}}, \\
\left\|\mathbf{W}_{i}^{\diamond} \mathbf{G}_{i}^{\sharp}\right\|_{F}^{2} \leq \frac{P^{\diamond}}{P_{t}},
\end{array}
$$

where $\overline{P^{\diamond}}=P^{\diamond} / P_{t}, \bar{\sigma}_{R_{i}}^{2}=\sigma_{R_{i}}^{2} / P_{t}$, and $\bar{P}_{R_{i}}=P_{R_{i}} / P_{t}$. Defining

$$
\begin{aligned}
c_{i} & =\operatorname{vec}\left(\mathbf{G}_{i}^{\diamond}\right)^{\dagger} \widetilde{\mathbf{W}}_{i} \operatorname{vec}\left(\mathbf{G}_{i}^{\diamond}\right)-\overline{P^{\diamond}}, \\
\widetilde{\mathbf{W}}_{i} & =\left(\left(\mathbf{W}_{i}^{\diamond}\right)^{\dagger} \mathbf{W}_{i}^{\diamond}\right) \odot \mathbf{I},
\end{aligned}
$$

the constraint (28) can be reformulated as

$$
\begin{aligned}
& \operatorname{vec}\left(\Delta_{\mathbf{G}_{i}}^{\dagger}\right) \widetilde{\mathbf{W}}_{i} \operatorname{vec}\left(\Delta_{\mathbf{G}_{i}}\right) \\
& +\Re\left\{\operatorname{vec}\left(\Delta_{\mathbf{G}_{i}}^{\dagger}\right) \widetilde{\mathbf{W}}_{i} \operatorname{vec}\left(\mathbf{G}_{i}^{\diamond}\right)\right\}+c_{i} \leq 0,
\end{aligned}
$$

which can be converted to the following LMI:

$$
\left[\begin{array}{cc}
\zeta_{i} \mathbf{I}_{N}-\widetilde{\mathbf{W}}_{i} & -\widetilde{\mathbf{W}}_{i} \operatorname{vec}\left(\mathbf{G}_{i}^{\diamond}\right) \\
-\operatorname{vec}\left(\mathbf{G}_{i}^{\diamond}\right)^{\dagger} \widetilde{\mathbf{W}}_{i}^{\dagger} & c_{i}-\zeta_{i} \alpha_{i}
\end{array}\right] \geq 0,
$$

where $\zeta_{i}$ is a slack variable.

Thus, the objective problem $Q_{2}$ is recast as

$$
\begin{aligned}
Q_{3}: \min _{\mathbf{B}, \mathbf{D}} & \gamma_{1}+\gamma_{2} \\
\text { s.t. } & \operatorname{tr}\left\{\mathbf{B B}^{\dagger}\right\} \leq P_{t}, \\
& \operatorname{tr}\left\{\mathbf{D D}^{\dagger}\right\} \leq P_{d},
\end{aligned}
$$

(23), (23), (31) hold, $\forall \zeta_{i}, \forall \epsilon_{i}, \forall \varsigma_{i}$.

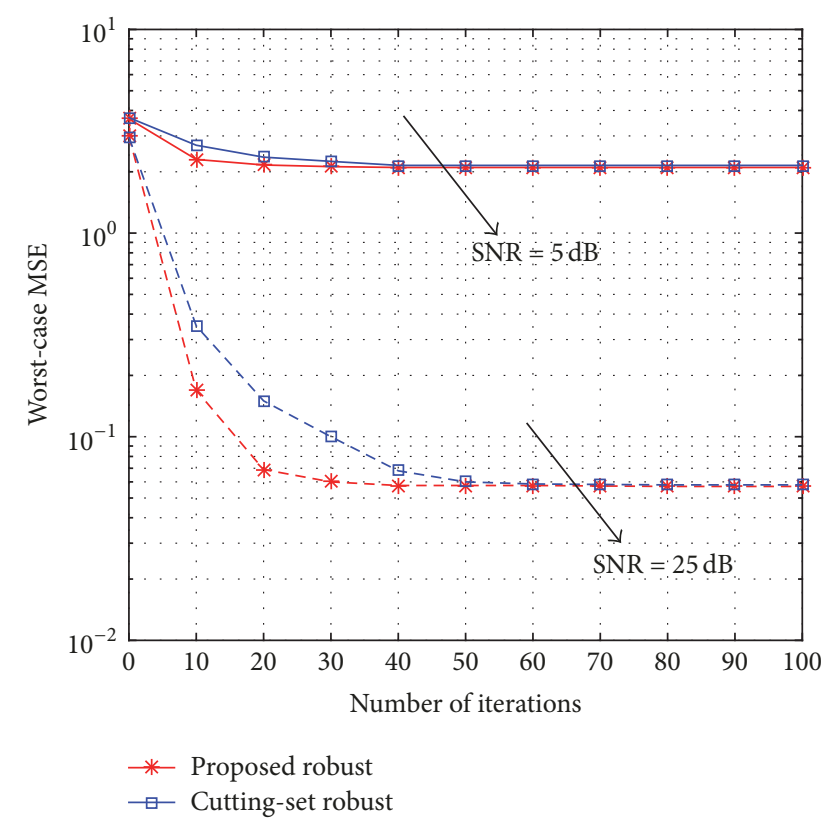

FIgURE 2: The worst-case MSE versus the number of iterations.

It is clear that the problem $Q_{3}$ is a biconvex SDP which can be efficiently solved by the following alternating algorithm by using CVX [27].

By initializing the small $\varphi$ and setting the limitation of the number of iterations $N_{\max }$, the suboptimal solution can be obtained when $\sum_{t=1}^{2} \gamma_{t}^{(n)}-\sum_{t=1}^{2} \gamma_{t}^{(n-1)} \leq \varphi$.

\section{Numerical Results}

In this section, we examine the performance of the proposed robust scheme in terms of the worst-case MSE compared with cutting-set method in [26], the nonrobust case and the perfect design. All results are averaged over 5,000 channel realizations and the initial BS and the UE beamforming matrices are chosen at random. We set the number of the relay nodes $L=3$, the number of antennas $M=4$ and $N=5$, respectively. The transmission power at the $\mathrm{BS}$ and the relay node is $P_{t}=P_{r}=1$. In the iterative algorithm, $\varphi$ and $N_{\max }$ are set to $10^{-3}$ and 100 , respectively.

Figure 2 depicts the convergence of the proposed algorithm with the fixed NBEs as $\alpha_{i}=\beta_{i}=0.01$ for the transmission SNR $=5 \mathrm{~dB}$ and $\mathrm{SNR}=25 \mathrm{~dB}$. Clearly, our proposed scheme has satisfactory convergent performance compared with the cutting-set method. It is because, in our proposed scheme, the objective problem converts the worst-case MSE into the one with $\gamma_{1}+\gamma_{2}$ which significantly reduces the computational complexity. In addition, the optimal (local) relay beamforming matrix can be efficiently derived which supports the practical utility of our design. It is also observed that, for the higher transmission SNR, the convergence rate of our proposed scheme outperforms the cutting-set one while both these two schemes provide the optimal (local) solution with more iterations. 


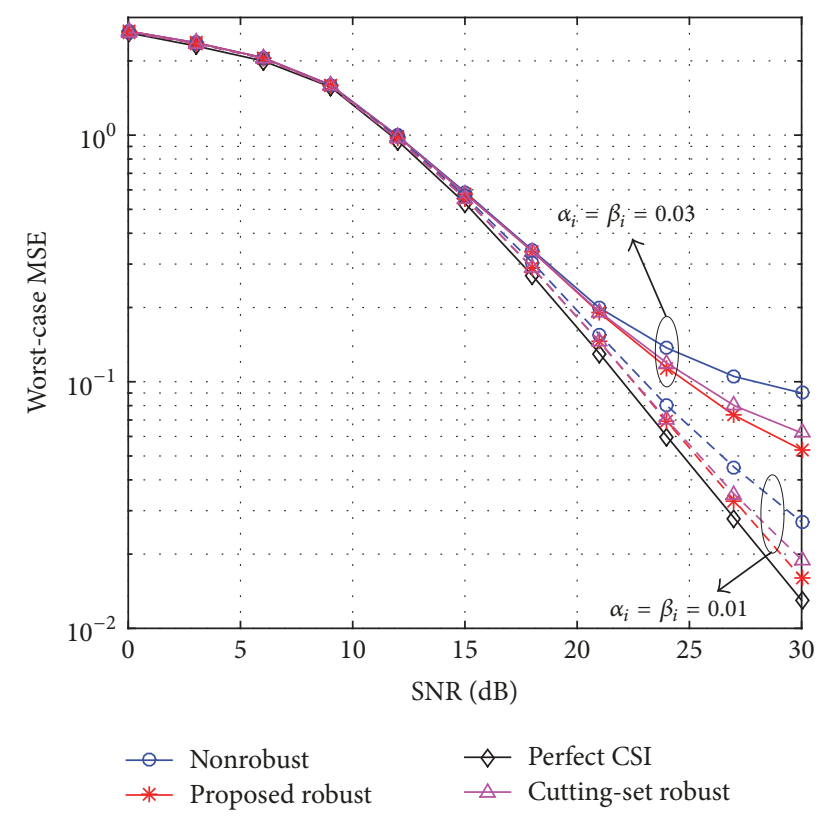

FIGURE 3: The worst-case MSE versus the transmission SNR.

Figure 3 compares the worst-case MSE in cases of our proposed scheme, the cutting-set one and the perfect one versus the transmission SNR with $\alpha_{i}=\beta_{i}=0.01$ and $\alpha_{i}=$ $\beta_{i}=0.03$, where the perfect one is served as the upper bound with the NBEs as $\alpha_{i}=\beta_{i}=0$ while the robust solutions are obtained by using the corresponding algorithms. Results reveal that our proposal has a relative advantage over the other two schemes, especially for the larger NBE case. This is reasonable, since employing the approximation may lose the performance, in our proposed scheme, the objective problem $Q_{3}$ is equivalent to the original target one without using the approximations (except the terms involving the high-order channel uncertainties) which is different to the work in [26].

\section{Conclusions}

So far, we have presented an efficient way to achieve signal transmission from a BS to an isolated, out-of-coverage user by realizing a generalized BS-R-UE beamforming. In our proposed cooperative MIMO multirelay networks with imperfect CSI; first, the optimal relay beamforming is derived as a means to improve the efficiency. Then, the semi-infinite objective problem is converted into a biconvex problem, which is subject to the LMI constraints. Furthermore, the converted, biconvex problem is efficiently solved by our alternating algorithm. The system performance in terms of worstcase MSE is verified using a set of qualitative analysis; the results show us that the proposed beamforming method outperforms the conventional schemes and can also effectively reduce the computational complexity when it is compared to the cutting-set schemes and also to the nonrobust ones. Our future concerns will be the generalized optimization problems on the two-way relay networks and cooperative relaying nonorthogonal multiple access (NOMA) systems.

\section{Conflicts of Interest}

The authors declare that they have no conflicts of interest.

\section{Acknowledgments}

The authors would gratefully acknowledge the grants from the National Natural Science Foundation of China (61371113, 61401241, 61401240, and 61771264), Nantong UniversityNantong Joint Research Center for Intelligent Information Technology (KFKT2016B01 and KFKT2017B01), the Open Research Fund of National Mobile Communications Research Laboratory, Southeast University (no. 2015D02), and the Brain Korea BK21 plus.

\section{References}

[1] H. Xie, F. Gao, and S. Jin, "An Overview of Low-Rank Channel Estimation for Massive MIMO Systems," IEEE Access, vol. 4, pp. 7313-7321, 2016.

[2] Z. Zhao, M. Peng, Z. Ding, W. Wang, and H.-H. Chen, "Denoise-and-forward network coding for two-way relay MIMO systems," IEEE Transactions on Vehicular Technology, vol. 63, no. 2, pp. 775-788, 2014.

[3] J. Fan, L. Li, H. Zhang, and W. Chen, "Denoise-and-Forward Two-Path Successive Relaying with DBPSK Modulation," IEEE Wireless Communications Letters, vol. 6, no. 1, pp. 42-45, 2017.

[4] M. Masjedi, A. M. Doost Hoseini, and S. Gazor, "NonCoherent Detection and Denoise-and-Forward Two-Way Relay Networks," IEEE Transactions on Communications, vol. 64, no. 11, pp. 4497-4505, 2016.

[5] H. X. Nguyen, N. N. Tran, and H. T. Nguyen, "Performance analysis of adaptive decode-and-forward relaying in noncoherent cooperative networks," EURASIP Journal on Wireless Communications and Networking, vol. 2013, no. 1, article no. 281, 2013.

[6] Q. Cui, T. Yuan, X. Tao, A. A. Dowhuszko, and R. Jantti, “Energy efficiency analysis of two-way DF relay system with non-ideal power amplifiers," IEEE Communications Letters, vol. 18, no. 7, pp. 1254-1257, 2014.

[7] C. Wang, H. Chen, Q. Yin, A. Feng, and A. F. Molisch, "Multiuser two-way relay networks with distributed beamforming," IEEE Transactions on Wireless Communications, vol. 10, no. 10, pp. 3460-3471, 2011.

[8] Y. Rong, "Joint source and relay optimization for two-way linear non-regenerative MIMO relay communications," IEEE Transactions on Signal Processing, vol. 60, no. 12, pp. 6533-6546, 2012.

[9] V. Havary-Nassab, S. Shahbazpanahi, and A. Grami, "Optimal distributed beamforming for two-way relay networks," IEEE Transactions on Signal Processing, vol. 58, no. 3, part 1, pp. 1238$1250,2010$.

[10] K. An, M. Lin, J. Ouyang, and H. Wei, "Performance analysis of beamforming in two-hop AF relay networks with antenna correlation and interference," AEÜ - International Journal of Electronics and Communications, vol. 68, no. 7, pp. 587-594, 2014.

[11] J. Joung and J. Choi, "Linear Precoder Design for an AF TwoWay MIMO Relay Node with No Source Node Precoding," IEEE Transactions on Vehicular Technology, 2017. 
[12] R. Budhiraja and B. Ramamurthi, "Transceiver Design for Nonconcurrent Two-Way MIMO AF Relaying with QoS Guarantees," IEEE Transactions on Vehicular Technology, vol. 65, no. 12, pp. 9651-9661, 2016.

[13] J.-M. Kang and H.-M. Kim, “Training Designs for Estimation of Spatially Correlated Fading Channels in MIMO Amplify-andForward Two-Way Multi-Relay Networks," IEEE Communications Letters, vol. 20, no. 4, pp. 772-775, 2016.

[14] C. Hu and Y. Chiu, "Robust joint optimization for MIMO AF multiple-relay systems with correlated channel uncertainties," International Journal of Electronics and Communications, vol. 68, no. 5, pp. 453-464, 2014.

[15] H. Tang, W. Chen, and J. Li, "Achieving Optimality in Robust Joint Optimization of Linear Transceiver Design," IEEE Transactions on Vehicular Technology, vol. 65, no. 3, pp. 1814-1819, 2016.

[16] K. Cumanan, Z. Ding, Y. Rahulamathavan, M. M. Molu, and H.H. Chen, "Robust MMSE beamforming for multiantenna relay networks," IEEE Transactions on Vehicular Technology, vol. 66, no. 5, pp. 3900-3912, 2017.

[17] G. Zhang, X. Li, M. Cui, G. Li, and Y. Tan, “Transceiver design for cognitive multi-user MIMO multi-relay networks using imperfect CSI," AEÜ - International Journal of Electronics and Communications, vol. 70, no. 5, pp. 544-557, 2016.

[18] L. Li, Y. Xu, Z. Chen, and J. Fang, "Robust transmit design for secure AF relay networks with imperfect CSI," EURASIP Journal on Wireless Communications and Networking, vol. 2016, no. 1, article no. 142, 2016.

[19] H. Shen, W. Xu, J. Wang, and C. Zhao, "A worst-case robust beamforming design for multi-antenna AF relaying," IEEE Communications Letters, vol. 17, no. 4, pp. 713-716, 2013.

[20] N. Lee, H. J. Yang, and J. Chun, "Achievable sum-rate maximizing af relay beamforming scheme in two-way relay channels," in Proceedings of the ICC 2008 - 2008 IEEE International Conference on Communications Workshops, pp. 300-305, China, May 2008.

[21] E. A. Gharavol and E. G. Larsson, "The sign-definiteness lemma and its applications to robust transceiver optimization for multiuser MIMO systems," IEEE Transactions on Signal Processing, vol. 61, no. 2, pp. 238-252, 2013.

[22] P. Ubaidulla and S. Aissa, "Robust two-way cognitive relaying: Precoder designs under interference constraints and imperfect CSI," IEEE Transactions on Wireless Communications, vol. 13, no. 5, pp. 2478-2489, 2014.

[23] B. K. Chalise and L. Vandendorpe, "MIMO relay design for multipoint-to-multipoint communications with imperfect channel state information," IEEE Transactions on Signal Processing, vol. 57, no. 7, pp. 2785-2796, 2009.

[24] S. A. Vorobyov, A. B. Gershman, and Z.-Q. Luo, "Robust adaptive beamforming using worst-case performance optimization: a solution to the signal mismatch problem," IEEE Transactions on Signal Processing, vol. 51, no. 2, pp. 313-324, 2003.

[25] W. Duan, M. Wen, X. Jiang, Y. Yan, and M. Ho Lee, "Sumrate maximization and robust beamforming design for MIMO two-way relay networks with reciprocal and imperfect CSI," EURASIP Journal on Wireless Communications and Networking, vol. 2016, no. 1, article no. 157, 2016.

[26] J. Liu, F. Gao, and Z. Qiu, "Robust transceiver design for downlink multiuser MIMO AF relay systems," IEEE Transactions on Wireless Communications, vol. 14, no. 4, pp. 2218-2231, 2015.

[27] S. Boyd and L. Vandenberghe, Convex Optimization, Cambridge University Press, Cambridge, UK, 2004. 


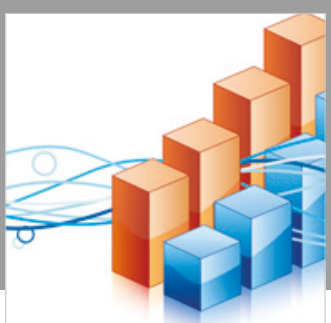

Advances in

Operations Research

\section{-n-m}
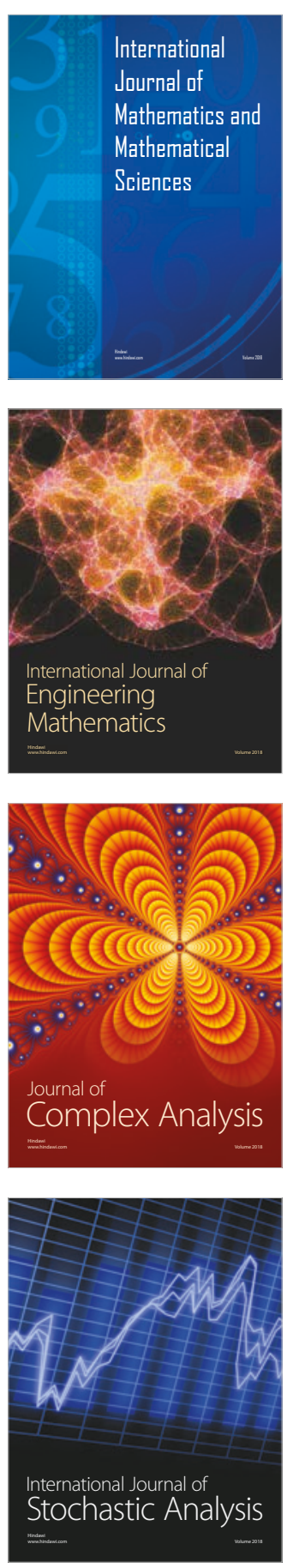
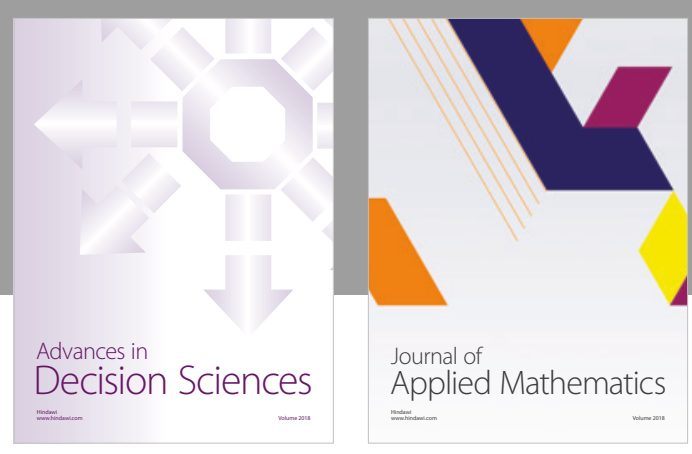

Journal of

Applied Mathematics
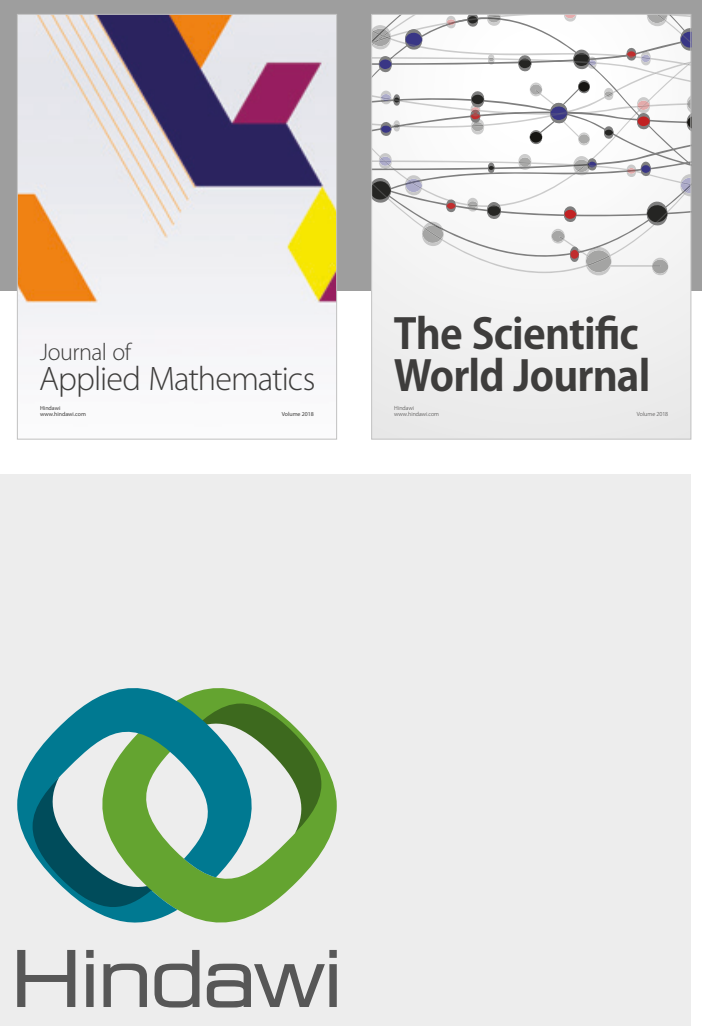

Submit your manuscripts at

www.hindawi.com

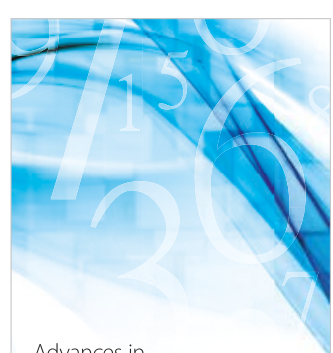

Advances in
Numerical Analysis
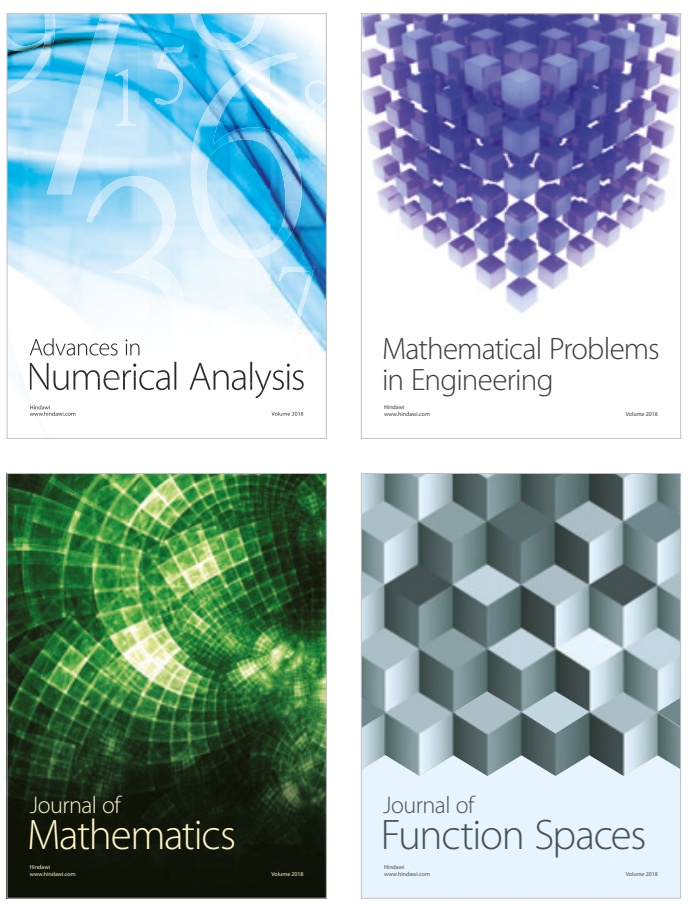

Mathematical Problems in Engineering

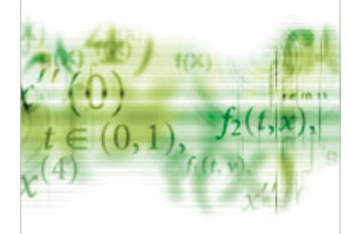

International Journal of

Differential Equations

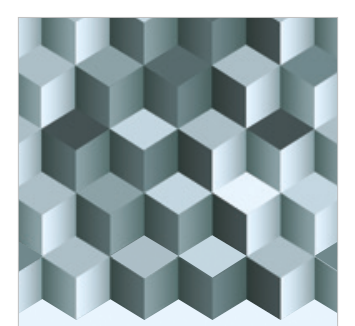

Journal of

Function Spaces

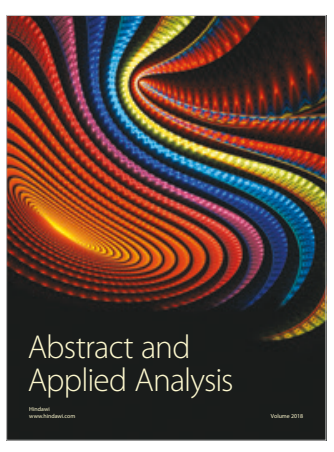

The Scientific

World Journal

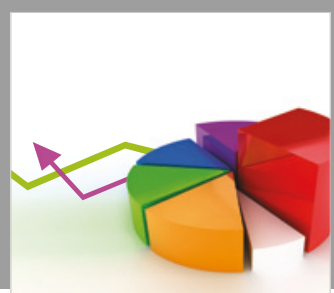

Journal of

Probability and Statistics
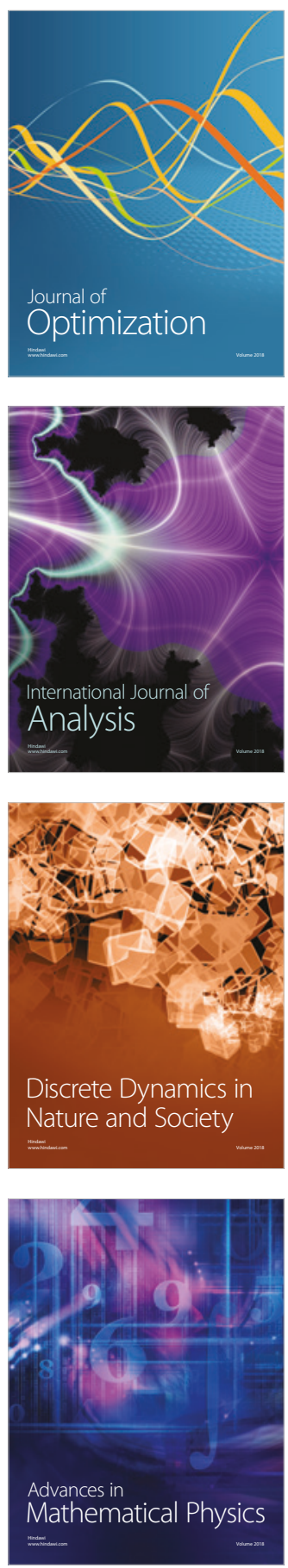